\title{
Upping Brightness 1000-Fold
}

\author{
By changing the material commonly used to make devices for generating \\ entangled photons, researchers create a quantum light source that is \\ significantly brighter than others.
}

\section{By Katherine Wright}

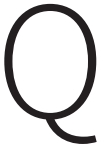
uantum technologies such as quantum computers require sources of entangled pairs of photons. To make these photon pairs, researchers commonly use silicon-based devices, where the silicon sits on an insulator. Galan Moody and his colleagues at the University of California, Santa Barbara, wondered if they could switch the silicon with aluminum gallium arsenide (AlGaAs), a material that has recently been demonstrated to be useful for classical photonics applications. The team showed that AlGaAs-made devices can produce entangled photons at a rate 1000 times greater than devices made from other materials [1].

Chip-sized devices for producing entangled pairs of photons typically couple laser light into a photonic waveguide or an optical cavity. Individual photons in the laser are then annihilated by interacting with the cavity's material, a process that creates two new-entangled-photons. The efficiency of this process depends on the size of the cavity and the properties of the material used to make it.

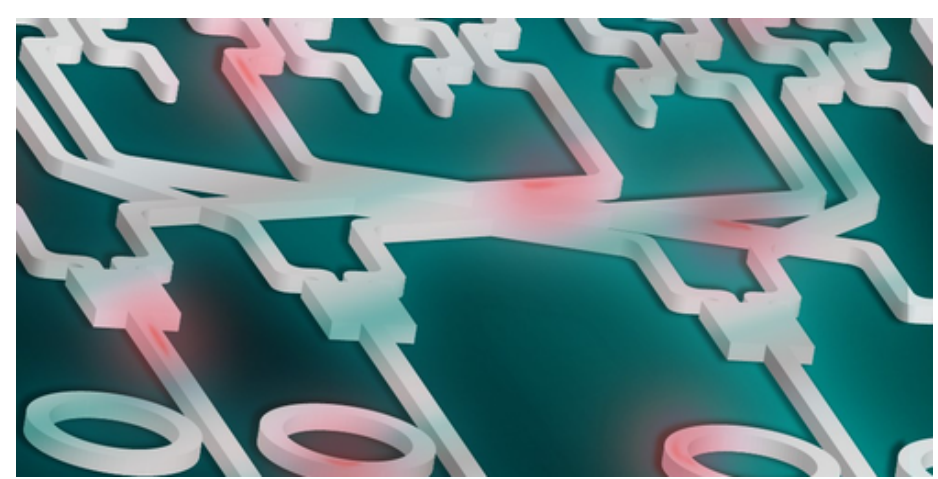

Credit: L. McKinney/Univ. California, Santa Barbara
Using this same design, Moody and his colleagues tinkered with the cavity's size and switched its material from silicon to AlGaAs. They found that they could increase the efficiency of the process significantly without compromising other important properties of the generated entangled photon pairs, such as entanglement fidelity.

The 1000-fold increase in brightness that the team achieved should, they say, let them produce the same number of entangled photon pairs as other devices but by using lower-power lasers. That could allow the modified devices to consume less power overall. AlGaAs could also be used to make the other components for quantum computers, potentially allowing them to be contained on a single chip. The team says that such integration would simplify device fabrication and facilitate all-on-chip quantum-photonics systems.

Katherine Wright is the Deputy Editor for Physics.

\section{REFERENCES}

1. T. J. Steiner et al., "Ultrabright entangled-photon-pair generation from an AlGaAs-on-insulator microring resonator," PRX Quantum 2, 010337 (2021). 